\title{
U-shaped sacral fracture: An easily missed fracture with high morbidity. A report of two cases.
}

\begin{abstract}
U-shaped sacral fracture is a very rare injury. This injury is easily missed and the diagnosis is often delayed as it is difficult to detect on the anteroposterior view of the pelvic radiograph. It is highly unstable and neurological injury is common. Two cases of U-shaped sacral fractures are reported here in which the diagnosis was delayed resulting in the late development of cauda equina syndrome. In these two cases, full recoveries were achieved following surgical decompression. A high index of suspicion with proper clinical and radiographic assessments will decrease the incidence of missed diagnosis and prevent the occurrence of delayed neurological deficits.
\end{abstract}

Keyword: U-shaped sacral fracture 